\title{
Modeling surface imperfections in thin films and nanostructured surfaces
}

\author{
Hansen, Poul-Erik; Madsen, J. S.; Jensen, S. A.; Madsen, M. H.; Karamehmedovi, Mirza
}

Published in:

Proceedings of SPIE

Link to article, DOI:

$10.1117 / 12.2270232$

Publication date:

2017

Document Version

Publisher's PDF, also known as Version of record

Link back to DTU Orbit

Citation (APA):

Hansen, P-E., Madsen, J. S., Jensen, S. A., Madsen, M. H., \& Karamehmedovi, M. (2017). Modeling surface imperfections in thin films and nanostructured surfaces. In Proceedings of SPIE (Vol. 10330). [103300J ] SPIE International Society for Optical Engineering. Proceedings of SPIE - The International Society for Optical Engineering https://doi.org/10.1117/12.2270232

\section{General rights}

Copyright and moral rights for the publications made accessible in the public portal are retained by the authors and/or other copyright owners and it is a condition of accessing publications that users recognise and abide by the legal requirements associated with these rights.

- Users may download and print one copy of any publication from the public portal for the purpose of private study or research.

- You may not further distribute the material or use it for any profit-making activity or commercial gain

- You may freely distribute the URL identifying the publication in the public portal 


\section{Modeling surface imperfections in thin films and nanostructured surfaces}

P.-E. Hansen

J. S. Madsen

S. A. Jensen

M. H. Madsen

M. Karamehmedovic 


\title{
Modeling surface imperfections in thin films and nanostructured surfaces
}

\section{P.-E. Hansen ${ }^{1}$, J. S. Madsen ${ }^{1,2}$, S. A. Jensen ${ }^{1}$, M. H. Madsen ${ }^{1}$, and M. Karamehmedovic ${ }^{3}$}

\author{
${ }^{1}$ Danish Fundamental Metrology A/S, Matematiktorvet 307, 2800 Kgs. Lyngby, Denmark \\ ${ }^{2}$ Center for Quantum Devices, Niels Bohr Institute, Universitetsparken 5, 2100 Copenhagen, \\ Denmark \\ ${ }^{3}$ Department of Applied Mathematics and Computer Science, Technical University of Denmark, \\ Kgs. Lyngby, DK-2800, Denmark \\ E-mail: peh@dfm.dk
}

\begin{abstract}
Accurate scatterometry and ellipsometry characterization of non-perfect thin films and nanostructured surfaces are challenging. Imperfections like surface roughness make the associated modelling and inverse problem solution difficult due to the lack of knowledge about the imperfection on the surface. Combining measurement data from several instruments increases the knowledge of non-perfect surfaces. In this paper, we investigate how to incorporate this knowledge of surface imperfection into inverse methods used in scatterometry and ellipsometry via Rigorous Coupled Wave Analysis. Three classes of imperfections are examined. The imperfections are introduced as periodic structures with super cell periods ten times larger than the simple grating period. Two classes of imperfections concern the grating and one class concern the substrate. It is shown that imperfections of a few nanometers can severely change the reflective response on silicon gratings. Inverse scatterometry analysis of gratings with imperfections using simulated data with white noise has been performed. The results show that scatterometry is a robust technology that is able to characterize grating imperfections provided that the imperfection class is known.
\end{abstract}

Introduction

In modern industry, there are growing needs for testing materials with micro- and nanometer structures that can reproduce fascinating effect from nature. These structures may be used to create structural color from diffraction or plasmonic effects without using paint, and therefore lowering the impact on the environment [1]. These structures are usually examined using atomic force microscopy, scanning electron microscopy or spectroscopic scatterometry [2]. Of these methods only spectroscopic scatterometry is capable of fast, in-line, nondestructive, noninvasive measurements [3]. However, spectroscopic scatterometry currently requires high quality structures and is therefore only widely used in the semi-conductor industry [4] . Hence, a noncontact method that can handle textured surfaces with roughness and defects would be attractive. Angular resolved scattering (ARS) is a light scattering method that has been proven to be able to analyze surfaces with nano- and micro roughness [5-7] as well as textured surfaces [12-14]. However, very little work has been done on combining the ability of ARS to study roughness and textured surfaces. In this paper, we present a theoretical study that examines the sensitivity of ARS to line gratings with defects and roughness. 
The ARS signal for a grating may be mathematically expressed in the following way [15]:

$$
A R S=\frac{P_{m}}{P_{i}} \frac{1}{\Omega}=\frac{1}{\lambda \cos \left(\theta_{m}\right)} \delta Q P S D(1)
$$

Where $P_{s} / P_{i}$ is the diffracted efficiency, $\Omega$ the solid angle through which the scattered light is detected, $\lambda$ is the wavelength of the light, $\theta_{m}$ is the diffraction angle, $\delta$ is the delta function that has a value of infinity at the diffraction angles and 0 otherwise, $Q$ is the polarization dependent reflection coefficient for the smooth interface and PSD is the power spectral density of the grating structure. The PSD function provides information about the actual grating shape which may include defect(s) and roughness of the surface. Equation (1) clearly demonstrates the relationship between the actual grating shape and the ARS (diffracted efficiency) signal.

Periodically placed objects form a diffraction grating [8]. When light encounters such a grating, the light is diffraction into different angles dictated by the grating equation:

$$
\Delta\left[\sin \left(\theta_{i}\right)+\sin \left(\theta_{m}\right)\right]=m \lambda
$$

Where $\Delta$ is the period of the grating, $\theta_{i}$ is the angle of incidence. The parameters are sketched in figure 1 . Other grating parameters, like the grating height and line width dictates how the reflecting light is coupled into different orders. These diffraction orders can therefore be seen as an optical fingerprint of the grating. This is utilized in scatterometry, where the topology of a grating is reconstructed from an optical response using an inverse modelling approach.

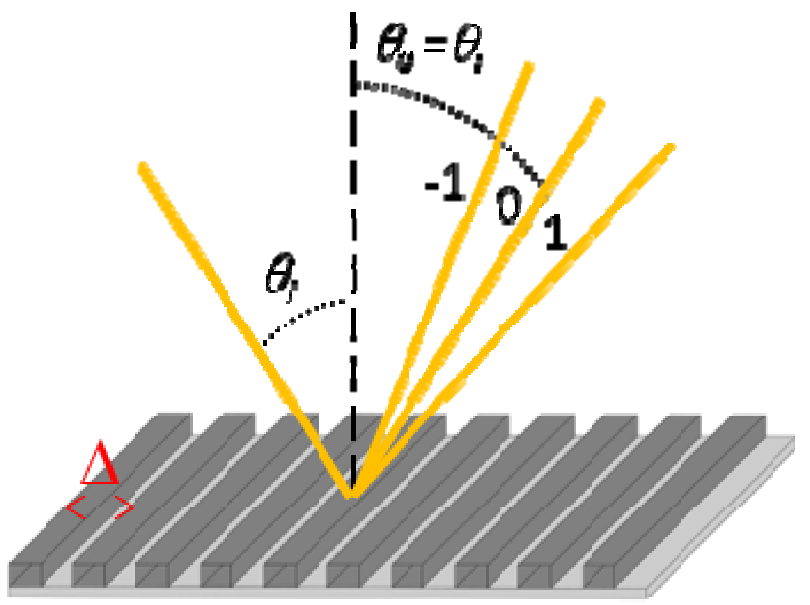

Figure 1. Schematic drawing of parameters in the grating equation.

\section{Simulations}

The simulations are based on rigorous coupled wave analysis, RCWA, where periodic structures are divided into rectangular slabs using a staircase approximation [9], see figure 2. As light encounters different media, the phase changes. This change in phase is used to determine how the structure changes the field reflected by the structure [10]. All modelled structures are based on perfect line gratings in silicon. The line gratings are described by their period $\Delta$, height $\mathrm{h}$, and their width $\mathrm{w}$. The simulated gratings have the following parameters: $\Delta=2 \mu \mathrm{m}, \mathrm{h}=0.7 \mu \mathrm{m}$ and $\mathrm{w}=$ $1 \mu \mathrm{m}$. In addition, imperfections are added to theses gratings. This is done by describing the grating as a super cell consisting of ten unit cells of the perfect grating, thus this super cell has a period, $\Gamma$, of $20 \mu \mathrm{m}$. The height of the different lines within the supercell are changed to implement the defects of the grating. In RCWA the incoming-, reflected- and transmitted fields are expressed as a Fourier expansion in terms of the spatial harmonics [6]. Using the supercells, the Fourier coefficients are calculated for each line within the supercell, and the resulting reflected field from all lines in a supercell is found.

Three classes of imperfections are examined in this study. A simple defect where the first of the ten grating lines are higher than 
the others, see figure 2 (a). A sinusoidal defect in the height over the grating, where the change in the height goes as a sine function, see figure 2 (b). A perfect grating on a sinus shaped substrate, see figure 2 (c). The magnitude of the imperfection is described by the parameter $d$. $d$ varies from $2 \%$ to $10 \%$ of the grating height (14 $\mathrm{nm}$ to $70 \mathrm{~nm})$. Simulations are performed using incoming light polarized in the direction of the grating lines, with a wavelength, $\lambda$, of $550 \mathrm{~nm}$ and an illumination angle, $\theta_{i}$, ranging from $-89.5^{\circ}$ to $89.5^{\circ}$ with respect to the grating normal.
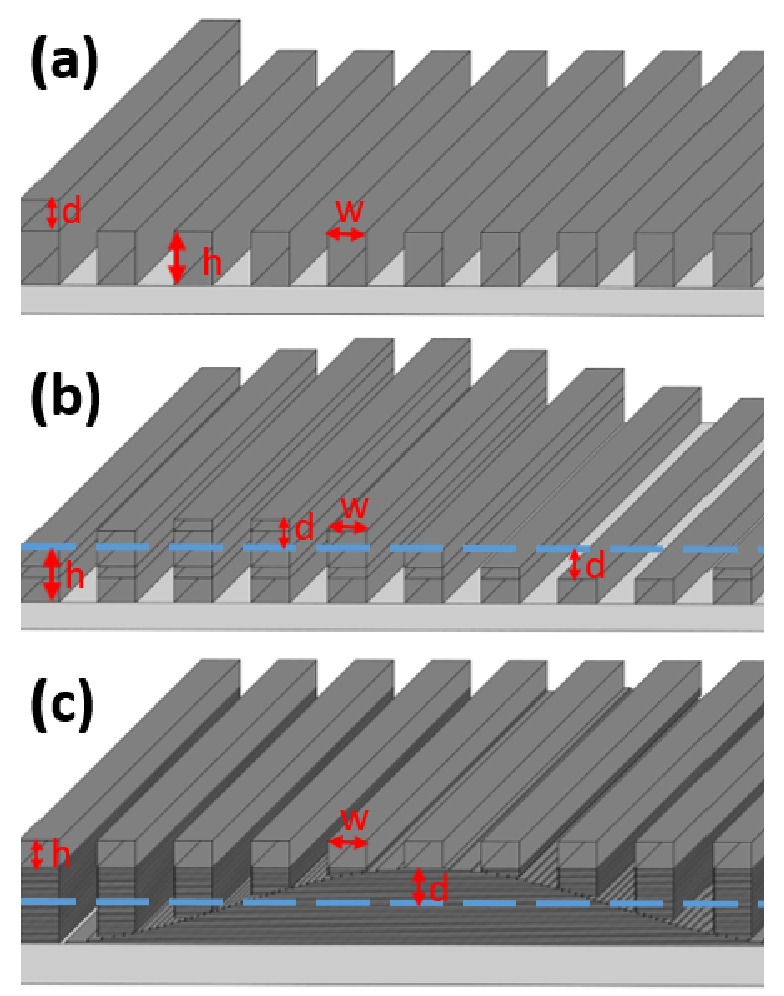

Figure 2. Sketches of the different grating imperfections examined. All sketches show a period of the supercell. The denoted parameters are the height of the perfect grating, h, the width of the grating, w, and the size of the imperfection, d. (a) Simple defect. (b) Sinusoidal defect. (c) A perfect grating on a sinusoidal substrate. All imperfections have been exaggerated for artistic purposes.

\section{Results}

In scatterometry one experimentally measures a diffraction efficiency, $\eta=\frac{P_{m}}{P_{i}}$, defined as the reflected light divided by the incoming light [3]. We define the change in diffraction efficiency, $\delta \eta$, caused by imperfections in the grating as:

$$
\delta \eta=\eta_{\text {Perfect }}-\eta_{\text {Defect }}
$$

Where $\eta_{\text {Perfect }}$ is the diffraction efficiency for a perfect grating and $\eta_{\text {Defect }}$ is the diffraction efficiency for a grating with the introduced defects. The changes in diffraction efficiency of the specular reflection for all simulated imperfections are shown in figure 3.

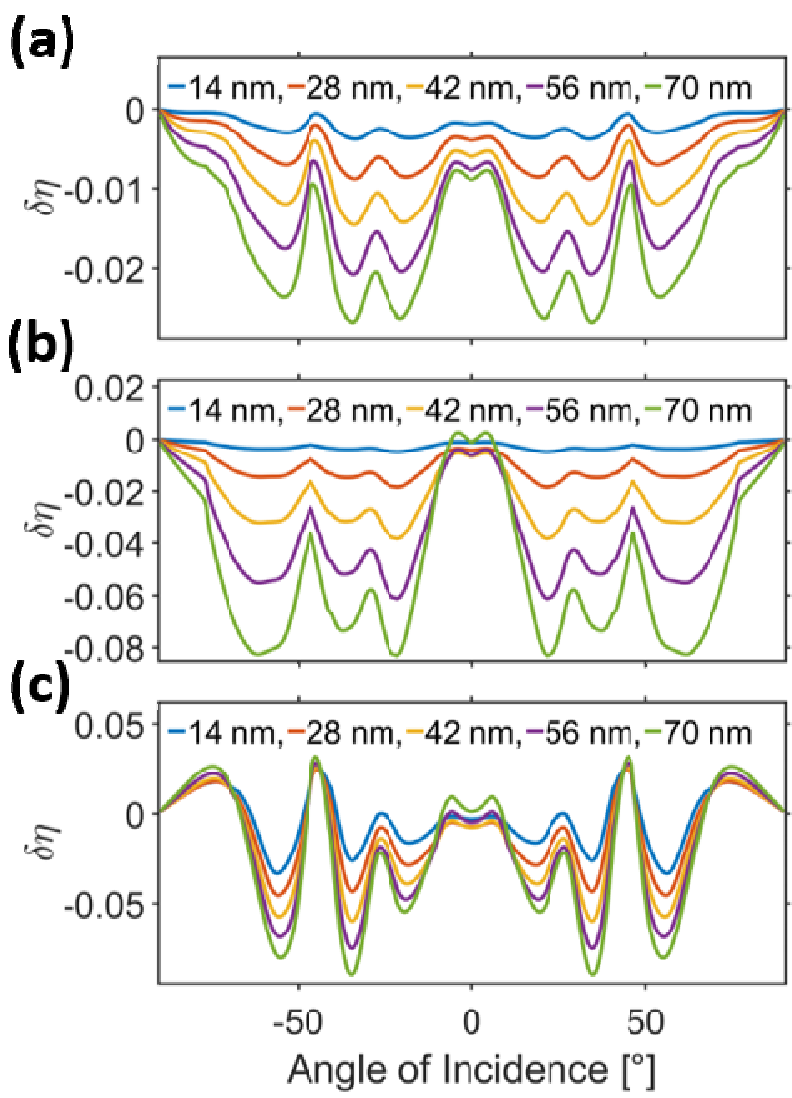

Figure 3. Changes in the specular reflection for the different imperfection classes. (a) Simple defect. (b) Sinusoidal grating defect. (c) Sinusoidal substrate defect. Note the different scales on the vertical axis. 
As a trend, the signal change is higher for larger imperfections, which is expected. The simple defect is found to have the lowest impact on the diffraction signal. This is not surprising, since only a tenth of the grating lines variy from the perfect grating. For the sinusoidal grating defect, the shape of the signal resembles the signal of the simple defect, however, the changes are much larger. For the largest magnitude of this defect class, we see that the reflection is amplified for small angles. Looking at the sinusoidal substrate defect, we see that the defects are impactful for all magnitudes. This shows that it is essential to know if the substrate contains periodic structures, rather than just looking at standard roughness measurements, if one desires an accurate reconstruction of grating parameters. For all magnitudes of this defect, we have several angles where the substrate amplifies the reflection.

In order to asses if the changes in the signal caused by the imperfections are detectable from a scatterometry measurement, white Guassian noise [11] is added to all the simulated signals, with a signal to noise ratio of $10 \mathrm{~dB}$. Mimicking a standard scatterometry procedure, the modified signals are then compared to all unmodified signals one at a time, as if they were a measured diffraction efficiency. This is done using a chi-square optimization given by:

$\chi^{2}=\frac{1}{N} \sum_{i=1}^{N}\left(\eta_{\text {Noise }}\left(\theta_{i}\right)-\eta_{\text {Simulated }}\left(\theta_{i}\right)\right)^{2}$

Where $N$ is the number of angles measured, $\eta_{\text {Noise }}$ is the modified signal, $\theta_{i}$, is the angle of incidence and $\eta_{\text {Simulated }}$ are the simulated diffraction efficiencies for all imperfection classes. The simulation with the lowest $\chi^{2}$ is found as the best solution

The modified signal of a sinusoidal grating defect with a magnitude of $42 \mathrm{~nm}$ and the best fitting simulation is seen in figure 4 .

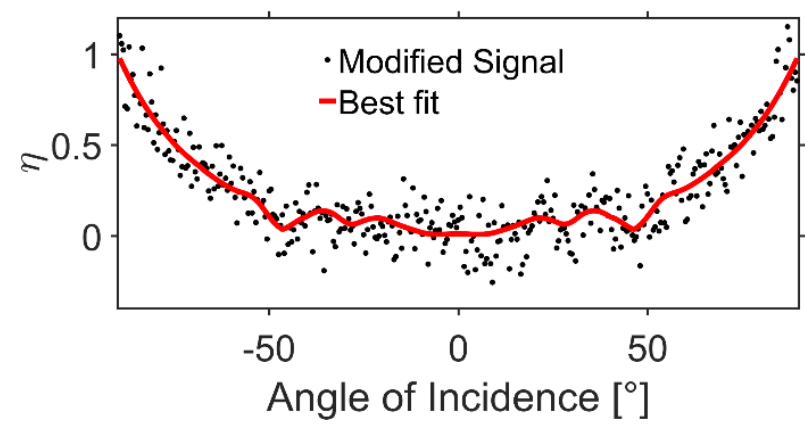

Figure 4. Signal from sinusoidal defect grating (magnitude $42 \mathrm{~nm}$ ) with added white Gaussian noise and the best fitting simulation. The best fitting model is found to be the original signal.

The chi-square optimization finds the original signal as the best fitting solution for all signals. This result demonstrates the robustness of the method and suggests that this approach can be used to detect imperfections on the grating or the substrate, provided one has an idea of the imperfections that could be present on the fabricated sample.

\section{Conclusion}

The influences of different types of imperfections on diffraction gratings have been examined by simulating their ARS signal. This has been done by describing imperfections in the grating as periodic structures with a larger period. Three classes have been investigated, two concerning the grating and one concerning the substrate. It is found that a change in the substrate has a higher impact than a height variation over the grating. Furthermore, an inverse scatterometry analysis is used to reconstruct the imperfections. The results suggest that grating imperfections can be characterized from the measured ARS signal, given that the imperfection class is known.

\section{Acknowledgements}

This work has received financial funding from the Eurostars project E8875 InFoScat and The Danish Agency for Science and Higher Education. 


\section{References}

[1] V.E. Johansen, L.H. Thamdrup, K. Smistrup, T. Nielsen, O. Sigmund, P. Vukusic, Designing visual appearance using a structured surface, Optica. 2 (2015) 239. doi:10.1364/OPTICA.2.000239.

[2] R.K. Leach, Fundamental principles of engineering nanometrology, Second edition, Elsevier, William Andrew, Amsterdam, 2014.

[3] M.H. Madsen, P.-E. Hansen, M. Zalkovskij, M. Karamehmedović, J. Garnæs, Fast characterization of moving samples with nano-textured surfaces, Optica. 2 (2015) 301. doi:10.1364/OPTICA.2.000301.

[4] C. Raymond, Overview Of Scatterometry Applications In High Volume Silicon Manufacturing, in: AIP, 2005: pp. 394-402. doi:10.1063/1.2062993.

[5] N. A Feidenhans'l, P-E. Hansen, L. Pilný, M. H Madsen, G. Bissacco, J. C. Petersen and R. Taboryski, Meas. Sci. Technol. 26 (2015) 08

[6] M.H. Madsen, P.-E. Hansen, Scatterometry - fast and robust measurements of nano-textured surfaces, Surf. Topogr. Metrol. Prop. 4 (2016) 023003. doi:10.1088/2051$672 \mathrm{X} / 4 / 2 / 023003$.

[7] J.C. Stover, Society of Photo-optical Instrumentation Engineers, Optical scattering: measurement and analysis, SPIE Optical Engineering Press, Bellingham, Wash., USA, 1995.

http://public.eblib.com/choice/publicfullrec ord.aspx?p=728552 (accessed February 6, 2017).

[8] T.D. Cope, The rittenhouse diffraction grating, J. Frankl. Inst. 214 (1932) 99-104. doi:10.1016/S0016-0032(32)90007-6.

[9] M.G. Moharam, T.K. Gaylord, D.A. Pommet, E.B. Grann, Stable implementation of the rigorous coupledwave analysis for surface-relief gratings: enhanced transmittance matrix approach, J. Opt. Soc. Am. A. 12 (1995) 1077. doi:10.1364/JOSAA.12.001077.

[10] P.-E. Hansen, L. Nielsen, Combined optimization and hybrid scalar-vector diffraction method for grating topography parameters determination, Mater. Sci. Eng. B. 165 (2009) 165-168. doi:10.1016/j.mseb.2009.09.006.
[11] K. McClaning, T. Vito, Radio receiver design, Noble Pub. Corp, Atlanta, GA, 2000.

[12] M. Karamehmedović, P.-E. Hansen, K. Dirscherl, E. Karamehmedović and T. Wriedt. Opt. Express, Vol. 20 No 9 (21678), 2012.

[13] P.-E. Hansen, B. Bilenberg, Y. R. Shen and M. Karamehmedovic. Proceeding of $13^{\text {th }}$ International Conference on Metrology and Properties of Engineering Surfaces, 12-15 April 2011. Editor R. Leach and L. Blunt. ISBN 978-0946754-59-5

[14]_B. Bodermann, P.-E. Hansen, S. Burger, M.-AHenn, H. Gross, M. Bär, F. Scholze, J. Endres and M. Wurm (2012). SPIE Proc. 8466, 84660E

[15] E . L . Church and P . Z . Takacs in Handbook of optics, second edition, volume I, M. Bass Editor (1995) 